Cambridge University Press

978-0-521-84752-o - Boolean Models and Methods in Mathematics, Computer Science, and Engineering

Edited by Yves Crama and Peter L. Hammer

Frontmatter

More information

\title{
Boolean Models and Methods in Mathematics, Computer Science, and Engineering
}

This collection of papers presents a series of in-depth examinations of a variety of advanced topics related to Boolean functions and expressions. The chapters are written by some of the most prominent experts in their respective fields and cover topics ranging from algebra and propositional logic to learning theory, cryptography, computational complexity, electrical engineering, and reliability theory. Beyond the diversity of the questions raised and investigated in different chapters, a remarkable feature of the collection is the common thread created by the fundamental language, concepts, models, and tools provided by Boolean theory. Many readers will be surprised to discover the countless links between seemingly remote topics discussed in various chapters of the book. This text will help them draw on such connections to further their understanding of their own scientific discipline and to explore new avenues for research.

Dr. Yves Crama is Professor of Operations Research and Production Management and former Dean of the HEC Management School of the University of Liège, Belgium. $\mathrm{He}$ is widely recognized as a prominent expert in the field of Boolean functions, combinatorial optimization, and operations research, and he has coauthored more than 70 papers and 3 books on these subjects. Dr. Crama is a member of the editorial board of Discrete Optimization, Journal of Scheduling, and 4OR - The Quarterly Journal of the Belgian, French and Italian Operations Research Societies.

The late Peter L. Hammer (1936-2006) was a Professor of Operations Research, Mathematics, Computer Science, Management Science, and Information Systems at Rutgers University and the Director of the Rutgers University Center for Operations Research (RUTCOR). He was the founder and editor-in-chief of the journals Annals of Operations Research, Discrete Mathematics, Discrete Applied Mathematics, Discrete Optimization, and Electronic Notes in Discrete Mathematics. Dr. Hammer was the initiator of numerous pioneering investigations of the use of Boolean functions in operations research and related areas, of the theory of pseudo-Boolean functions, and of the logical analysis of data. He published more than 240 papers and 19 books on these topics. 


\section{Cambridge University Press}

978-0-521-84752-0 - Boolean Models and Methods in Mathematics, Computer Science, and Engineering

Edited by Yves Crama and Peter L. Hammer

Frontmatter

More information

\section{ENCYCLOPEDIA OF MATHEMATICS AND ITS APPLICATIONS}

\section{FOUNDING EDITOR G.-C. ROTA}

Editorial Board

R. Doran, P. Flajolet, M. Ismail, T.-Y. Lam, E. Lutwak

The titles below, and earlier volumes in the series, are available from booksellers or from Cambridge University Press at www.cambridge.org.

100 E. Olivieri and M. Eulália Vares Large Deviations and Metastability

101 A. Kushner, V. Lychagin and V. Rubtsov Contact Geometry and Nonlinear Differential Equations

102 L. W. Beineke and R. J. Wilson (eds.) with P. J. Cameron Topics in Algebraic Graph Theory

103 O. J. Staffans Well-Posed Linear Systems

104 J. M. Lewis, S. Lakshmivarahan and S. K. Dhall Dynamic Data Assimilation

105 M. Lothaire Applied Combinatorics on Words

106 A. Markoe Analytic Tomography

107 P. A. Martin Multiple Scattering

108 R. A. Brualdi Combinatorial Matrix Classes

109 J. M. Borwein and J. D. Vanderwerff Convex Functions

110 M.-J. Lai and L. L. Schumaker Spline Functions on Triangulations

111 R. T. Curtis Symmetric Generation of Groups

112 H. Salzmann et al. The Classical Fields

113 S. Peszat and J. Zabczyk Stochastic Partial Differential Equations with Lévy Noise

114 J. Beck Combinatorial Games

115 L. Barreira and Y. Pesin Nonuniform Hyperbolicity

116 D. Z. Arov and H. Dym J-Contractive Matrix Valued Functions and Related Topics

117 R. Glowinski, J.-L. Lions and J. He Exact and Approximate Controllability for Distributed Parameter Systems

118 A. A. Borovkov and K. A. Borovkov Asymptotic Analysis of Random Walks

119 M. Deza and M. Dutour Sikirić Geometry of Chemical Graphs

120 T. Nishiura Absolute Measurable Spaces

121 M. Prest Purity Spectra and Localisation

122 S. Khrushchev Orthogonal Polynomials and Continued Fractions

123 H. Nagamochi and T. Ibaraki Algorithmic Aspects of Graph Connectivity

124 F. W. King Hilbert Transforms I

125 F. W. King Hilbert Transforms II

126 O. Calin and D.-C. Chang Sub-Riemannian Geometry

127 M. Grabisch et al. Aggregation Functions

128 L. W. Beineke and R. J. Wilson (eds.) with J. L. Gross and T. W. Tucker Topics in Topological Graph Theory

129 J. Berstel, D. Perrin and C. Reutenauer Codes and Automata

130 T. G. Faticoni Modules over Endomorphism Rings

131 H. Morimoto Stochastic Control and Mathematical Modeling

132 G. Schmidt Relational Mathematics

133 P. Kornerup and D. W. Matula Finite Precision Numbers Systems and Arithmetic 
Cambridge University Press

978-0-521-84752-0 - Boolean Models and Methods in Mathematics, Computer Science, and Engineering

Edited by Yves Crama and Peter L. Hammer

Frontmatter

More information

ENCYCLOPEDIA OF MATHEMATICS AND ITS APPLICATIONS

\section{Boolean Models and Methods in Mathematics, Computer Science, and Engineering}

Edited by

YVES CRAMA

Université de Liège

PETER L. HAMMER 


\section{Cambridge University Press}

978-0-521-84752-0 - Boolean Models and Methods in Mathematics, Computer Science, and Engineering

\section{Edited by Yves Crama and Peter L. Hammer}

Frontmatter

More information

CAMBRIDGE UNIVERSITY PRESS

Cambridge, New York, Melbourne, Madrid, Cape Town, Singapore, São Paulo, Delhi, Dubai, Tokyo, Mexico City

Cambridge University Press

32 Avenue of the Americas, New York, NY 10013-2473, USA

www.cambridge.org

Information on this title: www.cambridge.org/9780521847520

(c) Yves Crama and Peter L. Hammer 2010

This publication is in copyright. Subject to statutory exception and to the provisions of relevant collective licensing agreements, no reproduction of any part may take place without the written permission of Cambridge University Press.

First published 2010

Printed in the United States of America

A catalog record for this publication is available from the British Library.

Library of Congress Cataloging in Publication data

Boolean models and methods in mathematics, computer science, and engineering / edited by Yves Crama, Peter L. Hammer.

p. $\quad$ cm. - (Encyclopedia of mathematics and its applications ; 134)

Includes bibliographical references and index.

ISBN 978-0-521-84752-0

1. Algebra, Boolean. 2. Probabilities. I. Crama, Yves, 1958

II. Hammer, P. L., 1936- III. Title. IV. Series.

QA10.3.B658 2010

511.3'24-dc22 2010017816

ISBN 978-0-521-84752-0 Hardback

Cambridge University Press has no responsibility for the persistence or accuracy of URLs for external or third-party Internet Web sites referred to in this publication and does not guarantee that any content on such Web sites is, or will remain, accurate or appropriate. 
Cambridge University Press

978-0-521-84752-0 - Boolean Models and Methods in Mathematics, Computer Science, and Engineering

Edited by Yves Crama and Peter L. Hammer

Frontmatter

More information

\section{Contents}

Preface

page vii

Introduction

ix

Acknowledgments

xiii

Contributors

$\mathrm{xv}$

Acronyms and Abbreviations

xvii

\section{Part I Algebraic Structures}

1 Compositions and Clones of Boolean Functions

Reinhard Pöschel and Ivo Rosenberg

2 Decomposition of Boolean Functions

Jan C. Bioch

\section{Part II Logic}

3 Proof Theory

Alasdair Urquhart

4 Probabilistic Analysis of Satisfiability Algorithms John Franco

5 Optimization Methods in Logic John Hooker

\section{Part III Learning Theory and Cryptography}

6 Probabilistic Learning and Boolean Functions

Martin Anthony

7 Learning Boolean Functions with Queries

Robert H. Sloan, Balázs Szörényi, and György Turán 
Cambridge University Press

978-0-521-84752-0 - Boolean Models and Methods in Mathematics, Computer Science, and Engineering

Edited by Yves Crama and Peter L. Hammer

Frontmatter

More information

8 Boolean Functions for Cryptography and

Error-Correcting Codes

Claude Carlet

9 Vectorial Boolean Functions for Cryptography

Claude Carlet

\section{Part IV Graph Representations and Efficient Computation Models}

10 Binary Decision Diagrams

Beate Bollig, Martin Sauerhoff, Detlef Sieling, and Ingo Wegener

11 Circuit Complexity

Matthias Krause and Ingo Wegener

12 Fourier Transforms and Threshold Circuit Complexity

Jehoshua Bruck

13 Neural Networks and Boolean Functions

Martin Anthony

14 Decision Lists and Related Classes of Boolean Functions

Martin Anthony

\section{Part V Applications in Engineering}

15 Hardware Equivalence and Property Verification

J.-H. Roland Jiang and Tiziano Villa

16 Synthesis of Multilevel Boolean Networks

Tiziano Villa, Robert K. Brayton, and Alberto L.

Sangiovanni-Vincentelli

17 Boolean Aspects of Network Reliability

Charles J. Colbourn 
Cambridge University Press

978-0-521-84752-0 - Boolean Models and Methods in Mathematics, Computer Science, and Engineering

Edited by Yves Crama and Peter L. Hammer

Frontmatter

More information

\section{Preface}

Boolean models and methods play a fundamental role in the analysis of a broad diversity of situations encountered in various branches of science.

The objective of this collection of papers is to highlight the role of Boolean theory in a number of such areas, ranging from algebra and propositional logic to learning theory, cryptography, computational complexity, electrical engineering, and reliability theory.

The chapters are written by some of the most prominent experts in their fields and are intended for advanced undergraduate or graduate students, as well as for researchers or engineers. Each chapter provides an introduction to the main questions investigated in a particular field of science, as well as an in-depth discussion of selected issues and a survey of numerous important or representative results. As such, the collection can be used in a variety of ways: some readers may simply skim some of the chapters in order to get the flavor of unfamiliar areas, whereas others may rely on them as authoritative references or as extensive surveys of fundamental results.

Beyond the diversity of the questions raised and investigated in different chapters, a remarkable feature of the collection is the presence of an "Ariane's thread" created by the common language, concepts, models, and tools of Boolean theory. Many readers will certainly be surprised to discover countless links between seemingly remote topics discussed in various chapters of the book. It is hoped that they will be able to draw on such connections to further their understanding of their own scientific disciplines and to explore new avenues for research.

The collection intends to be a useful companion and complement to the monograph by Yves Crama and Peter L. Hammer, Boolean Functions: Theory, Algorithms, and Applications. Cambridge University Press, Cambridge, U.K., 2010, which provides the basic concepts and theoretical background for much of the material handled here. 
Cambridge University Press

978-0-521-84752-0 - Boolean Models and Methods in Mathematics, Computer Science, and Engineering

Edited by Yves Crama and Peter L. Hammer

Frontmatter

More information

\section{Introduction}

The first part of the book, "Algebraic Structures," deals with compositions and decompositions of Boolean functions.

A set $F$ of Boolean functions is called complete if every Boolean function is a composition of functions from $F$; it is a clone if it is composition-closed and contains all projections. In 1921, E. L. Post found a completeness criterion, that is, a necessary and sufficient condition for a set $F$ of Boolean functions to be complete. Twenty years later, he gave a full description of the lattice of Boolean clones. Chapter 1, by Reinhard Pöschel and Ivo Rosenberg, provides an accessible and self-contained discussion of "Compositions and Clones of Boolean Functions" and of the classical results of Post.

Functional decomposition of Boolean functions was introduced in switching theory in the late 1950s. In Chapter 2, "Decomposition of Boolean Functions," Jan C. Bioch proposes a unified treatment of this topic. The chapter contains both a presentation of the main structural properties of modular decompositions and a discussion of the algorithmic aspects of decomposition.

Part II of the collection covers topics in logic, where Boolean models find their historical roots.

In Chapter 3, "Proof Theory," Alasdair Urquhart briefly describes the more important proof systems for propositional logic, including a discussion of equational calculus, of axiomatic proof systems, and of sequent calculus and resolution proofs. The author compares the relative computational efficiency of these different systems and concludes with a presentation of Haken's classical result that resolution proofs have exponential length for certain families of formulas.

The issue of the complexity of proof systems is further investigated by John Franco in Chapter 4, "Probabilistic Analysis of Satisfiability Algorithms." Central questions addressed in this chapter are: How efficient is a particular algorithm when applied to a random satisfiability instance? And what distinguishes "hard" from "easy" instances? Franco provides a thorough analysis of these questions, starting with a presentation of the basic probabilistic tools and models and covering advanced results based on a broad range of approaches. 
Cambridge University Press

978-0-521-84752-0 - Boolean Models and Methods in Mathematics, Computer Science, and

Engineering

Edited by Yves Crama and Peter L. Hammer

Frontmatter

More information

In Chapter 5, "Optimization Methods in Logic," John Hooker shows how mathematical programming methods can be applied to the solution of Boolean inference and satisfiability problems. This line of research relies on the interpretation of the logical symbols 0 and 1 as numbers, rather than meaningless symbols. It leads both to fruitful algorithmic approaches and to the identification of tractable classes of problems.

The remainder of the book is devoted to applications of Boolean models in various fields of computer science and engineering, starting with "Learning Theory and Cryptography" in Part III.

In Chapter 6, "Probabilistic Learning and Boolean Functions," Martin Anthony explains how an unknown Boolean function can be "correctly approximated," in a probabilistic sense, when the only available information is the value of the function on a random sample of points. Questions investigated here relate to the quality of the approximation that can be attained as a function of the sample size, and to the algorithmic complexity of computing the approximating function.

A different learning model is presented by Robert H. Sloan, Balázs Szörényi, and György Turán in Chapter 7, "Learning Boolean Functions with Queries." Here, the objective is to identify the unknown function exactly by asking questions about it. The efficiency of learning algorithms, in this context, depends on prior information available about the properties of the target function, about the type of representation that should be computed, about the nature of the queries that can be formulated, and so forth. Also, the notion of "efficiency" can be measured either by the number of queries required by the learning algorithm (information complexity) or by the total of amount of computational steps required by the algorithm (computational complexity). The chapter provides an introduction and surveys a large variety of results along these lines.

In Chapter 8, Claude Carlet provides a very complete overview of the use of "Boolean Functions for Cryptography and Error-Correcting Codes." Both cryptography and coding theory are fundamentally concerned with the transformation of binary strings into binary strings. It is only natural, therefore, that Boolean functions constitute a basic tool and object of study in these fields. Carlet discusses quality criteria that must be satisfied by error-correcting codes and by cryptographic functions (high algebraic degree, nonlinearity, balancedness, resiliency, immunity, etc.) and explains how these criteria relate to characteristics of Boolean functions and of their representations. He introduces several remarkable classes of functions such as bent functions, resilient functions, algebraically immune functions, and symmetric functions, and he explores the properties of these classes of functions with respect to the aforementioned criteria.

In Chapter 9, "Vectorial Boolean Functions for Cryptography," Carlet extends the discussion to functions with multiple outputs. Many of the notions introduced in Chapter 8 can be naturally generalized in this extended framework: families of representations, quality criteria, and special classes of functions are introduced and analyzed in a similar fashion. 
Cambridge University Press

978-0-521-84752-0 - Boolean Models and Methods in Mathematics, Computer Science, and

Engineering

Edited by Yves Crama and Peter L. Hammer

Frontmatter

More information

Part IV concentrates on "Graph Representations and Efficient Computation Models" for Boolean functions.

Beate Bollig, Martin Sauerhoff, Detlef Sieling, and the late Ingo Wegener discuss "Binary Decision Diagrams" (BDDs) in Chapter 10. A BDD for function $f$ is a directed acyclic graph representation of $f$ that allows efficient computation of the value of $f(x)$ at any point $x$. Different types of BDDs can be defined by placing restrictions on the underlying digraph, by allowing probabilistic choices, and so forth. Questions surveyed in Chapter 10 are, among others: What is the size of a smallest BDD representation of a given function? How can a BDD be efficiently generated? How difficult is it to solve certain problems on Boolean functions (satisfiability, minimization, etc.) when the input is represented as a BDD?

Matthias Krause and Ingo Wegener discuss a different type of graph representations in Chapter 11, "Circuit Complexity." Boolean circuits provide a convenient model for the hardware realization of Boolean functions. Krause and Wegener describe efficient circuits for simple arithmetic operations, such as addition and multiplication. Further, they investigate the possibility of realizing arbitrary functions by circuits with small size or small depth. Although lower bounds or upper bounds on these complexity measures can be derived under various assumptions on the structure of the circuit or on the properties of the function to be represented, the authors also underline the existence of many fundamental open questions on this challenging topic.

Fourier transforms are a powerful tool of classical analysis. More recently, they have also proved useful for the investigation of complex problems in discrete mathematics. In Chapter 12, "Fourier Transforms and Threshold Circuit Complexity," Jehoshua Bruck provides an introduction to the basic techniques of Fourier analysis as they apply to the investigation of Boolean functions and neural networks. He explains, in particular, how they can be used to derive bounds on the size of the weights and on the depth of Boolean circuits consisting of threshold units.

The topic of "Neural Networks and Boolean Functions" is taken up again by Martin Anthony in Chapter 13. The author focuses first on the number and on the properties of individual threshold units, which can be viewed as linear, as nonlinear, or as "delayed" (spiking) threshold Boolean functions. He next discusses the expressive power of feed-forward artificial neural networks made up of threshold units.

Martin Anthony considers yet another class of graph representations in Chapter 14, "Decision Lists and Related Classes of Boolean Functions." A decision list for function $f$ can be seen as a sequence of Boolean tests, the outcome of which determines the value of the function on a given point $x$. Every Boolean function can be represented as a decision list. However, when the type or the number of tests involved in the list is restricted, interesting subclasses of Boolean functions arise. Anthony investigates several such restrictions. He also considers the algorithmic complexity of problems on decision lists (recognition, learning, equivalence), 
Cambridge University Press

978-0-521-84752-0 - Boolean Models and Methods in Mathematics, Computer Science, and Engineering

Edited by Yves Crama and Peter L. Hammer

Frontmatter

More information

and he discusses various connections between threshold functions and decision lists.

The last part of the book focuses on "Applications in Engineering."

Since the 1950s, electrical engineering has provided a main impetus for the development of Boolean logic. In Chapter 15, J.-H. Roland Jiang and Tiziano Villa survey the use of Boolean methods for "Hardware Equivalence and Property Verification." A main objective, in this area of system design, is to verify that a synthesized digital circuit conforms to its intended design. The chapter introduces the reader to the problem of formal verification, examines the complexity of different versions of equivalence checking ("given two Boolean circuits, decide whether they are equivalent"), and describes approaches to this problem. For the solution of these engineering problems, the authors frequently refer to models and methods covered in earlier chapters of the book, such as satisfiability problems or binary decision diagrams.

In Chapter 16, Tiziano Villa, Robert K. Brayton, and Alberto L. SangiovanniVincentelli discuss the "Synthesis of Multilevel Boolean Networks." A multilevel representation of a Boolean function is a circuit representation, similar to those considered in Chapter 11 or in Chapter 13. From the engineering viewpoint, the objective of multilevel implementations is to minimize the physical area occupied by the circuit, to reduce its depth, to improve its testability, and so on. Villa, Brayton, and Sangiovanni-Vincentelli survey efficient heuristic approaches for the solution of these hard computational problems. They describe, in particular, factoring and division procedures that can be implemented in "divide-and-conquer" algorithms for multilevel synthesis.

The combinatorial structure of operating or failed states of a complex system can be reflected through a Boolean function, called the structure function of the system. The probability that the system operates is then simply the probability that the structure function takes value 1. In Chapter 17, Charles J. Colbourn explores in great detail the "Boolean Aspects of Network Reliability." He reviews several exact methods for reliability computations, based either on "orthogonalization" or decomposition, or on inclusion-exclusion and domination. He also explains the intimate, though insufficiently explored, connections between Boolean models and combinatorial simplicial complexes, as they arise in deriving bounds on system reliability. 
Cambridge University Press

978-0-521-84752-0 - Boolean Models and Methods in Mathematics, Computer Science, and Engineering

Edited by Yves Crama and Peter L. Hammer

Frontmatter

More information

\section{Acknowledgments}

The making of this book has been a long process, and it has benefited over the years from the help and advice provided by several individuals. The editors gratefully acknowledge the contribution of these colleagues to the success of the endeavor.

First and foremost, all chapter contributors are to be thanked for the quality of the material that they have delivered, as well as for their patience and understanding during the editorial process.

Several authors have contributed to the reviewing process by cross-reading each other's work. Additional reviews, suggestions, and comments on early versions of the chapters have been kindly provided by Endre Boros, Nadia Creignou, Tibor Hegedűs, Lisa Hellerstein, Toshi Ibaraki, Jörg Keller, Michel Minoux, Rolf Möhring, Vera Pless, Gabor Rudolf, Mike Saks, Winfrid Schneeweiss, and Ewald Speckenmeyer.

Special thanks are due to Endre Boros, who provided constant encouragement and tireless advice to the editors over the gestation period of the volume. Marty Golumbic gave a decisive push to the process by bringing most contributors together in Haifa, in January 2008, on the occasion of the first meeting on "Boolean Functions: Theory, Algorithms, and Applications." Terry Hart provided the efficient administrative assistance that allowed the editors to keep track of countless mail exchanges.

Finally, I must thank my mentor, colleague, and friend, Peter L. Hammer, for helping me launch this ambitious editorial project, many years ago. Unfortunately, Peter did not live to see the outcome of our joint efforts. I am sure that he would have loved it, and that he would have been very proud of this contribution to the dissemination of Boolean models and methods. 
Cambridge University Press

978-0-521-84752-0 - Boolean Models and Methods in Mathematics, Computer Science, and Engineering

Edited by Yves Crama and Peter L. Hammer

Frontmatter

More information

\section{Contributors}

\section{Martin Anthony}

Department of Mathematics

London School of Economics and

Political Science, UK

\section{Jan C. Bioch}

Department of Econometrics Erasmus University Rotterdam,

The Netherlands

\section{Beate Bollig}

Department of Computer Science Technische Universität Dortmund, Germany

\section{Robert K. Brayton}

Department of Electrical Engineering \& Computer Sciences

University of California at Berkeley, USA

\section{Jehoshua Bruck}

Computation and Neural Systems and Electrical Engineering

California Institute of Technology, USA

\section{Claude Carlet}

Department of Mathematics

University of Paris 8, France

\section{Charles J. Colbourn}

Computer Science and Engineering

Arizona State University, USA

\section{John Franco}

Department of Computer Science

University of Cincinnati, USA

\section{John Hooker}

Tepper School of Business

Carnegie Mellon University, USA

\section{J.-H. Roland Jiang}

Department of Electrical Engineering National Taiwan University, Taiwan

\section{Matthias Krause}

Theoretical Computer Science

Mannheim University, Germany

\section{Reinhard Pöschel}

Institut für Algebra

Technische Universität Dresden,

Germany

\section{Ivo Rosenberg}

Département de Mathématiques et de Statistique

Université de Montréal, Canada 
Cambridge University Press

978-0-521-84752-0 - Boolean Models and Methods in Mathematics, Computer Science, and Engineering

Edited by Yves Crama and Peter L. Hammer

Frontmatter

More information

Alberto L. Sangiovanni-Vincentelli

Department of Electrical Engineering

\& Computer Sciences

University of California at Berkeley, USA

\section{Martin Sauerhoff}

Department of Computer Science

Technische Universität Dortmund, Germany

\section{Detlef Sieling}

Department of Computer Science

Technische Universität Dortmund, Germany

\section{Robert H. Sloan}

Department of Computer Science University of Illinois at Chicago, USA

\section{Balázs Szörényi}

Hungarian Academy of Sciences

University of Szeged, Hungary

\section{György Turán}

Department of Mathematics, Statistics, and Computer Science

University of Illinois at Chicago, USA

\section{Alasdair Urquhart}

Department of Philosophy

University of Toronto, Canada

\section{Tiziano Villa}

Dipartimento d'Informatica

University of Verona, Italy

\section{Ingo Wegener ${ }^{\dagger}$}

Department of Computer Science

Technische Universität Dortmund,

Germany 
Cambridge University Press

978-0-521-84752-0 - Boolean Models and Methods in Mathematics, Computer Science, and Engineering

Edited by Yves Crama and Peter L. Hammer

Frontmatter

More information

\section{Acronyms and Abbreviations}

$\begin{array}{ll}\text { AB } & \text { almost bent } \\ \text { AIG } & \text { AND-Inverter graph } \\ \text { ANF } & \text { algebraic normal form } \\ \text { APN } & \text { almost perfect nonlinear } \\ \text { ATPG } & \text { Automatic Test Pattern Generation (p. 698) } \\ \text { BDD } & \text { binary decision diagram } \\ \text { BED } & \text { Boolean Expression Diagram } \\ \text { BMC } & \text { bounded model checking } \\ \text { BP } & \text { branching program } \\ \text { C-1-D } & \text { complete-1-distinguishability } \\ \text { CDMA } & \text { code division multiple access } \\ \text { CEC } & \text { combinational equivalence checking } \\ \text { CNF } & \text { conjunctive normal form } \\ \text { CQ } & \text { complete quadratic } \\ \text { CTL } & \text { computation tree logic } \\ \text { DD } & \text { decision diagram } \\ \text { DNF } & \text { disjunctive normal form } \\ \text { DPLL } & \text { Davis-Putnam-Logemann-Loveland } \\ \text { EDA } & \text { electronic design automation } \\ \text { FBDD } & \text { free binary decision diagram } \\ \text { FCSR } & \text { feedback with carry shift register } \\ \text { FFT } & \text { fast Fourier transform } \\ \text { FRAIG } & \text { Functionally Reduced AIG } \\ \text { FSM } & \text { finite-state machine } \\ \text { FSR } & \text { feedback shift register } \\ \text { GPS } & \text { generalized partial spread } \\ \text { HDL } & \text { hardware description language } \\ \text { HFSM } & \text { hardware finite-state machine } \\ \text { HSTG } & \text { hardware state transition graph } \\ \text { IBQ } & \text { incomplete boundary query } \\ & \\ & \end{array}$


Cambridge University Press

978-0-521-84752-0 - Boolean Models and Methods in Mathematics, Computer Science, and Engineering

Edited by Yves Crama and Peter L. Hammer

Frontmatter

More information

xviii

Acronyms and Abbreviations

LFSR linear feedback shift register

LP linear programming

LTL linear temporal logic

MTBDD multiterminal binary decision diagram

NNF numerical normal form

OBDD ordered binary decision diagram

PAC probably approximately correct

PBDD partitioned binary decision diagram

$P C \quad$ propagation criterion

QBF quantified Boolean formula

ROBDD reduced ordered binary decision diagram

RTL register-transfer level

SAC strict avalanche criterion

SAT satisfiability [not an acronym]

SBS stochastic binary system

SCC strongly connected component

SEC sequential equivalence checking

SEM sample error minimization

SOP sum-of-product

SQ statistical query

STG state transition graph

UBQ unreliable boundary query

UMC unbounded model checking

VC Vapnik-Chervonenkis

XBDD extended binary decision diagram 
Cambridge University Press

978-0-521-84752-0 - Boolean Models and Methods in Mathematics, Computer Science, and Engineering

Edited by Yves Crama and Peter L. Hammer

Frontmatter

More information

Boolean Models and Methods in Mathematics,

Computer Science, and Engineering 